\title{
1-(2,4-二氯苯基)-4-甲基-5-(4-氯苯基)吡唑-3-羧酸的新合成方法
}

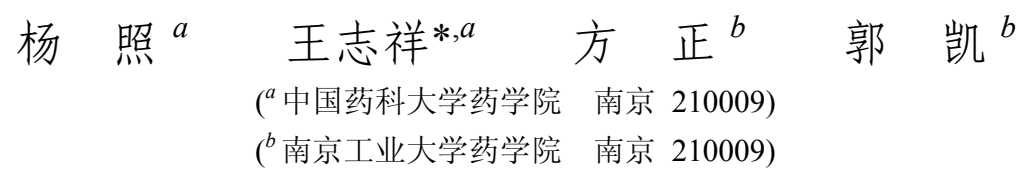

\begin{abstract}
摘要 研究了合成 1-(2,4-二氯苯基)-4-甲基-5-(4-氯苯基)吡唑-3-羧酸的新方法. 以价廉、易得的对氯苯甲醛为原料, 经 羟醛缩合、环合及氧化得目标化合物，合成总收率为 $50.6 \%$. 中间体及最终产物结构由 ${ }^{1} \mathrm{H} \mathrm{NMR,} \mathrm{MS}$ 确证. 该方法步骤 较少、操作简便、收率良好, 是一条适合中试放大的新合成工艺路线.

关键词 1,5-二芳基吡坐; 苯腙; 环合; 氧化; 合成
\end{abstract}

\section{A Novel Synthetic Method for 1-(2,4-Dichlorophenyl)-4-methyl- 5-(4-chlorophenyl)-1H-pyrazole-3-carboxylic Acid}

\author{
Yang, Zhao ${ }^{a} \quad$ Wang, Zhixiang*,a $\quad$ Fang, Zheng $^{b} \quad$ Guo, Kai $^{b}$ \\ $\left({ }^{a}\right.$ College of Pharmacy, China Pharmaceutical of University, Nanjing 210009) \\ ( ${ }^{b}$ School of Pharmaceutical Science, Nanjing University of Technology, Nanjing 210009)
}

\begin{abstract}
A novel method for the synthesis of 1-(2,4-dichlorophenyl)-4-methyl-5-(4-chlorophenyl)-1H-pyrazole-3-carboxylic acid was reported. The target compound was prepared starting from 4-chlorobenzaldehyde, which is inexpensive and easily available starting material, via adol condensation, cyclization and oxidation with the total yield of 50.6\%. The structures of intermediates and final product were determined by ${ }^{1} \mathrm{H}$ NMR and MS. The novel method with the advantages of shorten process, easily conducted procedure and relatively high yield is suitable for scale-up production.
\end{abstract}

Keywords 1,5-diarylpyrazole; phenyl hydrazone; cyclization; oxidation; synthesis

大麻素 1 (CB1)受体广泛分布于中枢神经系统和外 周组织器官, 在体内的作用主要包括食物摄取、能量平 衡、体内成份调节等综合作用 ${ }^{[1]}$. CB1 受体拮抗剂能够 有效减少机体对食物的摄取, 并增加能量的消耗, 对肥 胖症及相关的代谢综合症如 2 型糖尿病、脂代谢紊乱症 等有明显的预防和控制作用 ${ }^{[2]}$.

1-(2,4-二氯苯基)-4-甲基-5-(4-氯苯基)吡唑-3-羧酸 (1)是首个用于肥胖症治疗的 CB1 拮抗剂 Rimonabant ${ }^{[3]}$ 的关键中间体, 同时也可以用其合成一些其它具有 1,5二芳基吡唑结构的 $\mathrm{CB} 1$ 拮抗剂 ${ }^{[4]}$.

1 的合成报道 ${ }^{[5]}$ 较多, 但大都不适合工业化, 其中 具有一定中试潜力的路线主要是经 4 -氯苯丙酤(2)与三 甲基氯硅烷反应得烯醇硅醚 3,3 与草酸单酰氯单乙酯反 应得二酩酯 4,4 与 2,4-二氯苯肼缩合成苯腙 5 , 再于酸 催化下环合得吡唑羧酸酯 $\mathbf{6}$, 酯水解得 $\mathbf{1}$ (Scheme 1) ${ }^{[5 b]}$.
该路线所采用的原料、试剂廉价易得，但在实验室中难 以稳定重复出此工艺，可能原因是第一步产物 3 难以提 纯 (2 反应不完全), 在后续的步骤中带入较多杂质, 同 时中间体 3 6 均为油状物, 较难纯化导致反应不易中 控, 尽管 1 是固体, 但需要多次重结晶才能得到纯品, 另外有报道 ${ }^{[6]}$ 表明，合成 5 时可能导致异构体 7 的生成, 因此环合时不可避免产生副产物 8 (Scheme 2), 导致总 产率较低.

在参照文献[7]合成芳香吡唑羧酸化合物的基础上， 我们设计了一条新的合成 1 的路线, 以对氯苯甲醛(11) 为起始原料, 与硝基乙烷缩合得烯 12,12 与苯腙 10 (由 $D$-葡萄糖与 2,4-二氯苯胇缩合制得)环合成吡唑 13,13 再于碱性条件下氧化得 1 (Scheme 3). 新的合成方法步 骤较短, 操作简单, 适合放大生产.

* E-mail: zhixwang@163.com

Received October 17, 2012; revised November 23, 2012; published online November 26, 2012.

Project supported by the National Key Basic Research Program of China (973 Program) (Nos. 2012CB725204, 2012CB721104).

国家重点基础研究发展计划(973 计划, Nos. 2012CB725204, 2012CB721104)资助项目. 


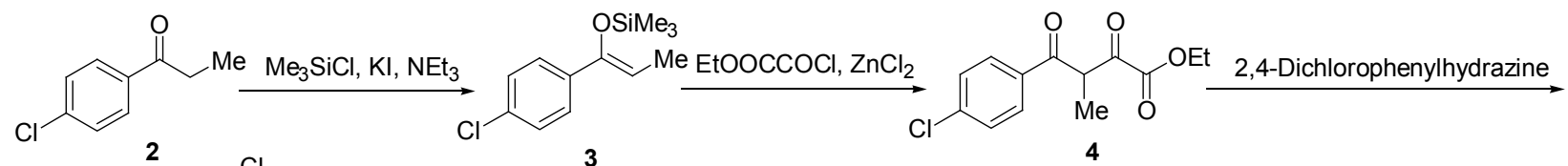<smiles>CCOC(=O)C(C)C(=NNc1ccc(Cl)cc1Cl)C(=O)c1ccc(Cl)cc1</smiles><smiles>CCOC(=O)c1nn(-c2ccc(Cl)cc2Cl)c(-c2ccc(Cl)cc2)c1C(O)(O)OOC(=O)c1c(C(=O)O)nn(-c2ccc(Cl)cc2Cl)c1-c1ccc(Cl)cc1</smiles>

Scheme 1<smiles>CCOC(=O)C(=O)C(C)C(=O)c1ccc(Cl)cc1</smiles>

Scheme 2<smiles>O=C[C@H](O)[C@@H](O)[C@@H](O)[C@H](O)CO</smiles>

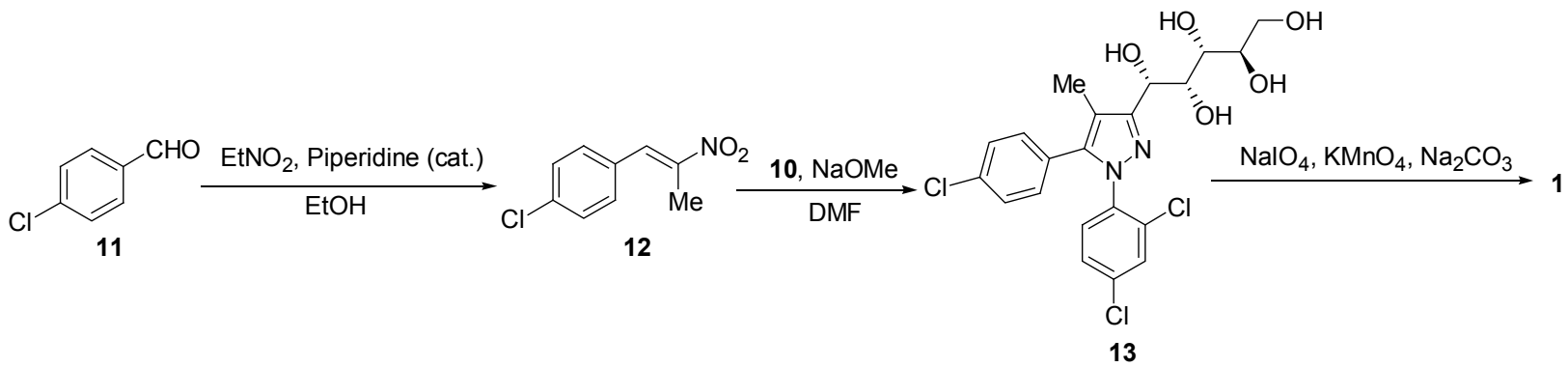

Scheme 3

\section{1 结果与讨论}

目前常用的合成 1 的方法是将对氯苯丙酮(2)经烯 醇化后, 合成得到二酮酯化合物 4, 4 再与苯肼缩合后环 合、水解. 但用三甲基氯硅烷烯醇化转化率仅能达 $80 \%{ }^{[5 b]}$ ，未反应原料无法纯化，将一直带入后续反应中， 也可采用六甲基二硅基胺基锂制成锂盐 ${ }^{[5 b]}$, 但成本较 高且操作繁琐, 另外, 缩合一步中不可避免出现的异构 体 7, 给后续的操作带来不便, 导致总产率难以提高. 因此, 改用对氯苯甲醛为原料的路线, 避免了上述副反 应的发生, 产物比较单一, 具有工艺化潜力.

合成 13 的反应中, 可能的机理是苯腙 10 中连接苯
基的 $\mathrm{N}$ 对 12 中的硝基烯发生加成反应，再环合得到 13 (Scheme 4). 考察了不同碱性试剂 $(\mathrm{KOH}, \mathrm{MeONa}$, 二异 丙基乙二胺和吡啶)和催化量酸性试剂(HAc 和 TsOH)对 反应的影响, 通过 TLC 监测发现, 仅 MeONa 能够发生 反应，而其它试剂几乎不反应，说明需要较强的碱性试 剂才能够使该环合反应发生，同时甲醇钠中 $\mathrm{Na}$ 离子会 与化合物糖基中的羟基发生络合，该方式也可能对此反 应有促进作用.

文献[7]报道是将类似结构吡唑上的 3 位多元醇碳 链先经过高碘酸钠氧化成醛基, 然后醛基再经氧化银氧 化成羧基, 实际操作中, 高碘酸钠氧化成醛这步产率较 低，不易提纯，监控发现有继续氧化的羧酸副产物的生 


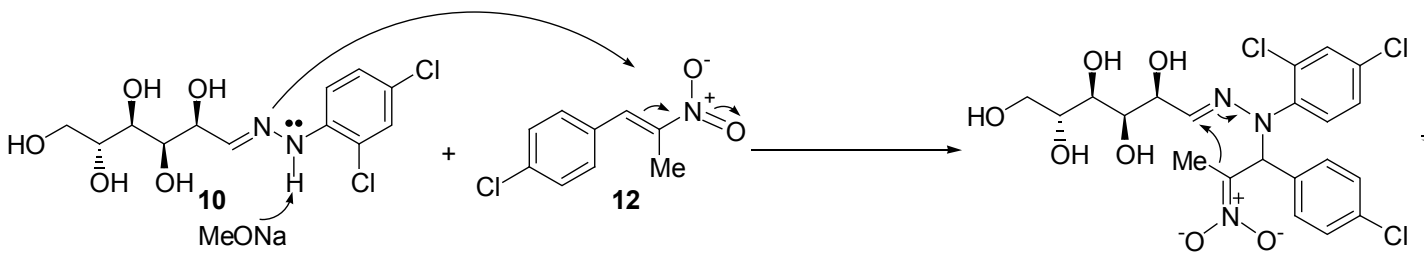

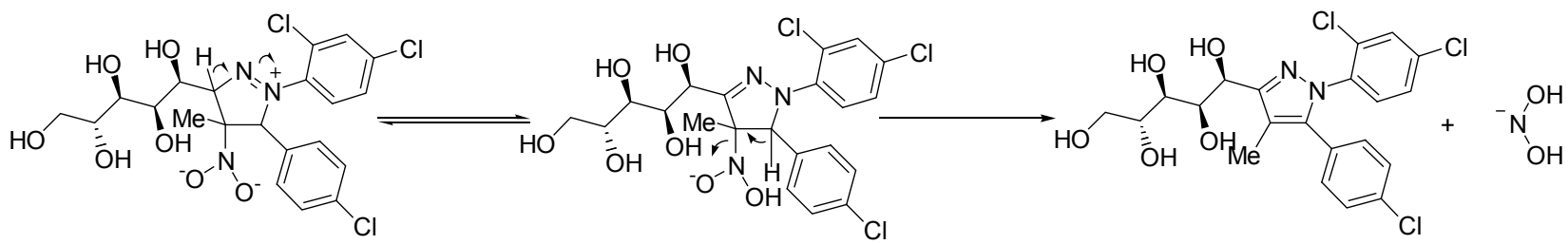

Scheme 4

成, 因此, 采用一步氧化法, 反应后生成的羧酸钠盐水 溶液经有机溶剂洗涤除去一部分杂质, 再酸化后得到 $\mathbf{1}$ 的粗品. 在文献基础上进行工艺优化后, 步骤缩短, 操 作简便, 且产率得到提高.

采用溶剂缓慢挥发培养目标物 1 晶体, 其单晶 $(0.30$ $\mathrm{mm} \times 0.24 \mathrm{~mm} \times 0.15 \mathrm{~mm}$ ) 在 R-AXIS SPIDER 衍射仪上, 于 $293 \mathrm{~K}$ 下用经石墨单色化的 MO K $\alpha$ 射线 $(\lambda=0.071071$ $\mathrm{nm}$ ) 在 $1.39^{\circ} \leqslant \theta \leqslant 25.27^{\circ}$ 范围内以 $\omega-2 \theta$ 扫描方式测得, 单晶结构见分子结构图(图 1), 晶体数据已上传至剑桥 晶体数据库(CCDC), 编号 712509 .

\section{2 结论}

本文研究了合成 1-(2,4-二氯苯基)-4-甲基-5-(4-氯苯 基)吡唑-3-羧酸的新方法, 用廉价易得的对氯苯甲醛经 三步反应，总收率可达 $50.6 \%$. 核磁、质谱表征了中间 体及目标化合物的结构. 合成方法操作简单, 后处理方 便, 具有中试放大潜力. 这种环合成吡唑及氧化成羧酸 的方法, 还可以方便的用于合成除 Rimonabant 以外的 具有 1,5-二芳基吡唑结构的 CB1 受体拮抗剂, 如 Surinabant, AM 251 等.

\section{3 实验部分}

\section{1 仪器与试剂}

采用 Aglilent 1100 LC/MS 质谱仪测定(ESI-MS); 采 用 Bruker AV-500 or AV-300 型核磁共振仪测定 ${ }^{1} \mathrm{H}$ NMR; 采用 YRT-3 熔点测定仪 (天津大学精密仪器厂) 测定中间体及产物的熔点; 采用 Rigaku Saturn 724 CCD $\mathrm{X}$-ray 单晶衍射仪测定单晶结构.

对氯苯甲醛(11)和 2,4-二氯苯肼盐酸盐为工业级, 其它试剂均为化学纯或分析纯.

\section{2 实验方法}

\subsubsection{D - 葡萄糖-2,4-二氯苯腙 $(\mathbf{1 0})$ 的合成}

$2,4-$ 二氯苯肼盐酸盐 $(21.3 \mathrm{~g}, 0.1 \mathrm{~mol})$ 的水 $(150 \mathrm{~mL})$ 溶液中加入醋酸钠 $(6.6 \mathrm{~g}, 0.1 \mathrm{~mol})$ 及 $D$-葡萄糖 $(36.0 \mathrm{~g}$, $0.2 \mathrm{~mol}), 25{ }^{\circ} \mathrm{C}$ 搅拌 $12 \mathrm{~h}$, 甲苯 $(30 \mathrm{~mL} \times 2)$ 洗涤溶液, 水 层用乙酸乙酯提取 $(50 \mathrm{~mL} \times 4)$, 合并乙酸乙酯层, 饱和 $\mathrm{NaCl}$ 溶液 $\left(50 \mathrm{~mL}\right.$ ) 洗涤有机层, 无水 $\mathrm{Na}_{2} \mathrm{SO}_{4}$ 干燥, 过滤, 浓缩, 得黄色固体 $26.5 \mathrm{~g}$, 产率 $78.2 \%{ }^{[8]}$. m.p. 143 148 ${ }^{\circ} \mathrm{C}$; ${ }^{1} \mathrm{H}$ NMR (DMSO- $d_{6}$ ) $\delta$ : $3.73 \sim 3.89(\mathrm{~m}, 5 \mathrm{H}, \mathrm{CH}$ ),

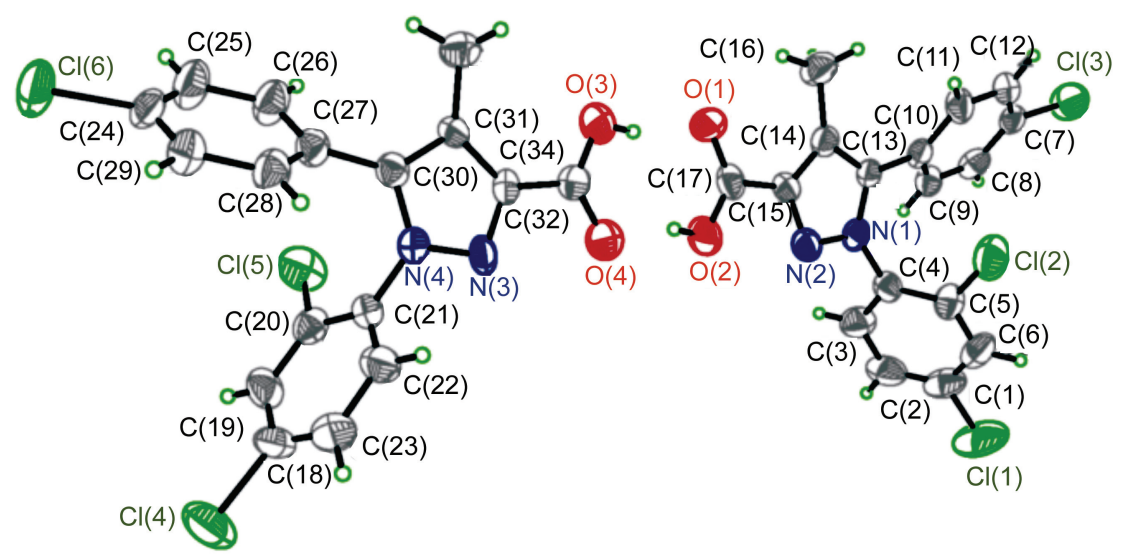

图 1 化合物 1 的分子结构图

Figure 1 Molecular structure of compound 1 
$4.01 \sim 4.03(\mathrm{~m}, 1 \mathrm{H}, \mathrm{CH}-\mathrm{OH}), 4.06(\mathrm{~s}, 1 \mathrm{H}, \mathrm{OH}), 4.72 \sim 4.79$ (m, 2H, OH), 5.03 (s, 1H, OH), $5.20(\mathrm{~s}, 1 \mathrm{H}, \mathrm{OH}), 7.03(\mathrm{~d}$, $J=6.9 \mathrm{~Hz}, 1 \mathrm{H}, \mathrm{N}=\mathrm{CH}), 7.55(\mathrm{dd}, J=8.4,2.1 \mathrm{~Hz}, 1 \mathrm{H}$, Ph-H), 7.68 (d, $J=8.4 \mathrm{~Hz}, 1 \mathrm{H}, \mathrm{Ph}-\mathrm{H}), 7.89$ (d, $J=2.1 \mathrm{~Hz}$, 1H, Ph-H), 9.69 (s, 1H, NH); ESI-MS m/z: $339.0[\mathrm{M}+$ $\mathrm{H}]^{+}$.

\subsubsection{1-对氯苯基-2-硝基丙烯(12)的合成}

对氯苯甲醛 $(11,14.1 \mathrm{~g}, 0.1 \mathrm{~mol})$ 的无水乙醇 $(100$ $\mathrm{mL})$ 溶液中加入硝基乙烷(8.6 mL, $0.12 \mathrm{~mol}$ )及哌啶 $(2$ 滴), 回流反应 $12 \mathrm{~h}$, 溶液浓缩至 $40 \mathrm{~mL}$, 静置析晶, 得 淡黄色片状结晶 $15.7 \mathrm{~g}$, 产率 79.2\% ${ }^{[9]}$. m.p. 85 86 ${ }^{\circ} \mathrm{C}$ [文献值 ${ }^{[9]}$ : m.p. 86 87 ${ }^{\circ} \mathrm{C}$ ]; ${ }^{1} \mathrm{H}$ NMR $\left(\mathrm{CDCl}_{3}\right) \delta: 2.44$ (s, 3H, $\left.\mathrm{CH}_{3}\right), 7.37$ (d, $\left.J=8.4 \mathrm{~Hz}, 2 \mathrm{H}, \mathrm{Ph}-\mathrm{H}\right), 7.43$ (d, $J=$ $8.4 \mathrm{~Hz}, 2 \mathrm{H}, \mathrm{Ph}-\mathrm{H}), 8.02(\mathrm{~s}, 1 \mathrm{H},=\mathrm{CH})$; ESI-MS $\mathrm{m} / \mathrm{z}$ : $198.0[\mathrm{M}+\mathrm{H}]^{+}$.

3.2.3 (1S,2R,3R,4R)-1-[1-(2,4-二氯苯基)-4- 甲基5-(4-氯苯基)吡唑-3-基]戊五醇(13)的合成

$12(1.97 \mathrm{~g}, 0.01 \mathrm{~mol})$ 和 $10(3.39 \mathrm{~g}, 0.01 \mathrm{~mol})$ 的无水 $N, N$-二甲基甲酰胺 $(20 \mathrm{~mL})$ 溶液中, 加入甲醇钠 $(0.54 \mathrm{~g}$, $0.01 \mathrm{~mol})$, 室温搅拌 $2 \mathrm{~d}$, 过滤除去少量不溶物, 减压蒸 除溶剂, 加乙酸乙酯 $(2 \mathrm{~mL})$ 研磨析出固体, 过滤得粗品, 甲醇重结晶得类白色固体 $3.65 \mathrm{~g}$, 收率 74.8\%. m.p. $196 \sim 199{ }^{\circ} \mathrm{C} ;{ }^{1} \mathrm{H}$ NMR (DMSO-d 6 ) $\delta: 2.21$ (s, 3H, $\mathrm{CH}_{3}$ ), $3.68 \sim 3.97(\mathrm{~m}, 7 \mathrm{H}, \mathrm{CH} \& \mathrm{OH}), 4.32 \sim 4.49(\mathrm{~m}, 3 \mathrm{H}, \mathrm{OH})$, 5.06 (s, 1H, OH), 7.29 (d, J=8.4 Hz, 2H, Ph-H), 7.44 (d, $J=8.4 \mathrm{~Hz}, 2 \mathrm{H}, \mathrm{Ph}-\mathrm{H}), 7.49$ (dd, $J=8.4,2.2 \mathrm{~Hz}, 1 \mathrm{H}$, Ph-H), 7.68 (d, $J=8.5 \mathrm{~Hz}, 1 \mathrm{H}, \mathrm{Ph}-\mathrm{H}), 7.72$ (d, $J=2.2 \mathrm{~Hz}$, $1 \mathrm{H}, \mathrm{Ph}-\mathrm{H}) ;{ }^{13} \mathrm{C}$ NMR (DMSO- $\left.d_{6}\right) \delta: 9.6\left(\mathrm{CH}_{3}\right), 63.9,71.6$, $72.4,72.9,73.2,118.1,126.9$ (×2), 127.4, 132.5, 133.0 $(\times 2), 133.6,134.2,134.7,135.3,136.2,138.0,141.3$, 153.1; HRMS (ESI) calcd for $\mathrm{C}_{21} \mathrm{H}_{21} \mathrm{Cl}_{3} \mathrm{~N}_{2} \mathrm{O}_{5}[\mathrm{M}+\mathrm{H}]^{+}$ 487.0589 , found 487.0580 .

3.2.4 1-(2,4-二氯苯基)-4-甲基-5-(4-氯苯基)吡唑-3翔酸 $(1)$ 的合成

$13(4.88 \mathrm{~g}, 0.01 \mathrm{~mol})$ 的叔丁醇 $(50 \mathrm{~mL})$ 悬浮液中, 加 入饱和碳酸钠溶液 $(20 \mathrm{~mL})$, 室温下搅拌滴加高碘酸钠 $(8.56 \mathrm{~g}, 0.04 \mathrm{~mol})$ 与高锰酸钾 $(79 \mathrm{mg}, 0.5 \mathrm{mmol})$ 的水 $(30$ $\mathrm{mL}$ )溶液, 滴加完毕后 $25{ }^{\circ} \mathrm{C}$ 反应 $3 \mathrm{~h}$, 升温至 $60{ }^{\circ} \mathrm{C}$ 反 应 $0.5 \mathrm{~h}$. 冷却至室温, 过滤除去不溶物, 用二氯甲烷 $(20 \mathrm{~mL})$ 洗涤, 水层用 $2 \mathrm{~mol} / \mathrm{L}$ 盐酸调节 $\mathrm{pH}$ 至 2 , 析出固 体, 过滤得白色固体粉末，80\%乙酸重结晶得白色结晶 3.26 g, 收率 $85.4 \%$. m.p. $209 \sim 212{ }^{\circ} \mathrm{C}$ [文献值 ${ }^{[5 b]}$ : m.p. $\left.210{ }^{\circ} \mathrm{C}\right] ;{ }^{1} \mathrm{H}$ NMR (DMSO- $\left.d_{6}\right) \delta: 2.23\left(\mathrm{~s}, 3 \mathrm{H}, \mathrm{CH}_{3}\right), 7.23$ (d, $J=8.4 \mathrm{~Hz}, 2 \mathrm{H}, \mathrm{Ph}-\mathrm{H}), 7.45$ (d, $J=8.4 \mathrm{~Hz}, 2 \mathrm{H}, \mathrm{Ph}-\mathrm{H})$, 7.57 (dd, $J=8.5,2.2 \mathrm{~Hz}, 1 \mathrm{H}, \mathrm{Ph}-\mathrm{H}), 7.70$ (d, $J=8.5 \mathrm{~Hz}$, 1H, Ph-H), 7.77 (d, J=2.2 Hz, 1H, Ph-H), 12.91 (br, 1H, $\mathrm{COOH}) ;{ }^{13} \mathrm{C}$ NMR (DMSO- $\left.d_{6}\right) \delta: 9.5,119.9,127.2,128.3$, 128.9 ( $\times 2), 131.4,131.8,132.5$ ( $\times 2), 133.6,135.2$, 134.6, 137.3, 143.6, 144.9, 165.3; ESI-MS $m / z$ : 381.0 $[\mathrm{M}+\mathrm{H}]^{+}$.

\section{References}

[1] (a) Munro, S.; Thomas, K. L.; Abu-Shaar, M. Nature 1993, 365, 61. (b) Kyrou, I.; Valsamakis, G.; Tsigos, C. Ann. N. Y. Acad. Sci. 2006, 1083, 270.

[2] Akbas, F.; Gasteyger, C.; Sjödin, A.; Astrup, A.; Larsen, T. M. Obes. Rev. 2009, 10, 58 .

[3] VanGaal, L. F.; Rissanen, A. M.; Scheen, A. J.; Ziegler, O.; Rossner, S. Lancet 2005, 365, 1387.

[4] (a) Vazquez, N.; Gomez-Vallejo, V.; Llop, J. Tetrahedron. Lett. 2012, 53, 4743.

(b) Tu, G. G.; Xiong, F.; Huang, H. M.; Kuang B. H.; Li, S. H. J. Enzyme Inhib. Med. Chem. 2011, 26, 222.

(c) Sasmal, P. K.; Reddy, D. S.; Talwar, R.; Venkatesham, B.; Balasubrahmanyam, D.; Kannan, M.; Srinivas, P.; Kumar, K. S.; Devi, B. N.; Jadhav, V. P.; Khan, S. K.; Mohan, P.; Chaudhury, H.; Bhuniya, D.; Iqbal, J.; Chakrabarti, R. Bioorg. Med. Chem. Lett. 2011, 21, 562.

(d) Wu, C.; Hung, M.; Song, J.; Yeh, T.; Chou, M.; Chu, C.; Jan, J.; Hsieh, M.; Tseng, S.; Chang, C.; Hsieh, W.; Lin, Y.; Yeh, Y.; Chung, W.; Kuo, C.; Lin, C.; Shy, H.; Chao, Y.; Shia, K. J. Med. Chem. 2009, 52, 4496.

[5] (a) Dutta, A. K.; Sard, H.; Ryan, W.; Razdan, R. K.; Compton, D. R.; Martin, B. R. Med. Chem. Res. 1995, 5, 54.

(b) Barth, F.; Casellas, P.; Congy, C.; Martinez, S.; Rinaldi, M.; Ann-Archard, G. EP 656354, 1994 [Chem. Abstr. 1994, 123, 286006].

(c) Tang, L.-H.; Tao, L.; Chen, H.-B.; Zhong, B.-H. Chin. J. Pharm. 2007, 38, 252 (in Chinese).

(汤立合，陶林，陈合兵，仲伯华，中国医药工业杂志，2007，38， 252.)

(d) Kotagiri, V. K.; Suthrapu, S.; Reddy, J. M.; Rao, C. P.; Bollugoddu, V.; Bhattacharya, A.; Bandichhor, R. Org. Process Res. Dev. 2007, 11, 910 .

(e) Nerdinger, S. WO 2008101860, 2008 [Chem. Abstr. 2008, 149, 307838].

(f) Jin, W.-H.; Zhang, P.-Z.; Ji, Y.-F. Chin. J. Synth. Chem. 2010, 18, 397 (in Chinese).

(金文虎, 张鹏志, 冀亚飞, 合成化学, 2010, 18, 397.)

[6] Donohue, S. R.; Halldin, C.; Pike, V. W. Tetrahedron Lett. 2008, 49, 2789.

[7] Gomez, G. M.; Conde Jimenez, J. L.; Podio Lora, V. An. Quim.; Ser. C 1986, 82, 204.

[8] Stroh, H. H.; Lamprecht, H. Chem. Ber. 1963, 96, 651.

[9] Foye, W. O. J. Pharm. Sci. 1979, 68, 591. 